\section{Über den gegenwärtigen Stand der Fetthärtung.}

Von Dr. W. NormanN, Emmerich (Rhein).

Vorgetragen auf der Hauptversammlung in Hamburg am 8 . Juni 1922 in der Hauptsitzung (Eingeg. 16./9. 1922.)

Nachdem wir auf unserer letzten Tagung vor dem Kriege in Bonn einen fesselnden Vortrag von B erg i u s über die damals noch ganz junge Industrie der Fetthärtung hören konnten, ist die Frage wohl berechtigt, wie sich diese Industrie bis heute entwickelt hat. Jch will versuchen, auf diese Frage Antwort zu geben, soweit dies möglìch ist.

Die Vorgeschichte der Fetthärtung ist so oft erörtert worden, daß ich deren Kenntnis wohl voraussetzen darf. Von den älteren Verfahren hat die Behandlung sehr schlechter Fette, insbesondere schlechten Knochenfettes, mit Schwefelsäure und nachfolgender Destillation ihren Platz in der Technik behauptet. Im übrigen kommt immer nur die katalytische Fetthärtung in Betracht.

Auch die Kenntnis dieser darf ich im allgemeinen wohl als bekannt voraussetzen; ich will nur daran erinnern, daß sie in der Anlagerung von Wasserstoff an die Doppelbindungen ungesättigter ole mit Hilfe eines Katalysators besteht, wodurch die flüssigen öle in die technisch viel wertvolleren festen Fette übergehen.

$$
\text { Technisches. }
$$

Utber den heutigen Stand der technischen Ausführungsformen der Fetthärtung sind wir einigermaßen gut unterrichtet durch die zahlreichen Patente, welche auf diesem Gebiete genommen worden sind und fortlaufend noch genommen werden. Allein in Deutschland sind etwa 100 Patente erteilt oder angemeldet. In den übrigen, auße:deutschen Ländern Europas wiederholt sich ein großer Teil der deutschen Patente, so daß die Gesamtziffer zwar das Vielfache der deutschen Anzahl betragen dürtte, ohne aber inhaltlich wesentlich Neues zu bringen. Dazu kommt noch eine große Zahl amerikanischer Patente, die in der Alten Welt nicht angemeldet sind; denn die in bezu? auf Fetthärtungspatente außerordentlich fruchtbaren Amerikaner verschmähen es meist, ihre Erfindungen auch in dem alten, faulen Europa anzumelden. Ich will mich bemühen, aus dieser Fülle von nur den Sonderlachmann interessierenden Einzelheiten einiges von allgemeinerem Interesse herauszuheben. Bei der Kürze der zur Verfügung stehenden Zeit ist auch hierin große Beschränkung notwendig.

Der Angelpunkt der ganzen Fetthärtung ist der Katalysator. Als solcher wird in der Praxis jetzt wohl ganz ausschließlich Nickel verwandt. Die Edelmetalle, die vorübergehend in geringem Umfange benutzt worden sind, sind jetzt ihrer Unerschwinglichkeit wegen ausgeschlossen. Kobalt und Kupfer werden für sich allein nicht angewandt. Mischungen dieser Metalle mit Nickel sind in Vorschlag gebracht; ob sie aber praktische Anwendung gefunden haben, ist mir nicht bekannt.

Als Ausgangsstoffe für die Nickelkatalysatoren werden beinahe sämtliche Nickelverbindungen vorgeschlagen, die es gibt, mit allen nur denkbaren Variationen in der Herstellung, und alle diese Katalysatoren haben nach Ansicht der Erfinder ganz überraschende, nicht vorauszusehende und hervorragende Eigenschaften, die sie allen anderen Nickelkatalysatoren selbstverständlich weit überlegen machen. Die Wahrheit ist: sie sind mehr oder weniger alle brauchbar. Die Art der Wirkung ist stets die gleiche; denn sie bestehen ja alle aus demselben Grundstoff. Kleine Unterschiede im Grade der Wirkung erklären sich aus der mehr oder weniger feinen Verteilung des Nickels, die aber nicht allein vom Verfahren der Herstellung, sondern auch von der Geschicklichkeit des Herstellers abhängt.

Das ursprüngliche Ausgangsmaterial war nach $\mathrm{S}$ a b a tiers Vorgang Nickeloxyd, welches durch Reduktion im Wasserstoffstrom in feinpulveriges Metall übergeführt wurde. Schon sehr bald, schon während der ersten Ausarbeitung einer Fabriksapparatur wurde erkannt, daß man die Wirkung des Nickels ganz bedeutend steigern kann, wenn man es nach dem Vorbilde anderer Katalysatoren auf Kieselgur als Träger niederschlägt. Man bewerkstelligt dieses Niederschlagen durch Fällen von Nickelsulfat mit Soda in Gegenwart von Kieselgur. Der Urheber dieses wertvollen Gedankens ist, wie ich gegenüber anderslautenden Angaben des Schrifttums feststellen möchte, der während des Krieges mehrfach genannte und namentlich von den heimkehrenden Englandgeiangenen mit Recht hochgepriesene Deutschengländer $\mathrm{Dr}$. $\mathrm{Markel}$ in London. Diese frühzeitige Verbesserung des Katalysators hatte zur Folge, daß der alte $\mathrm{Sa}$ bat i er sche Reinnickelkatalysator gar nicht erst in den Großbetrieb eingeführt $\mathrm{zu}$ werden brauchte. Hieraus ist gelegentlich der Schluß gezogen worden, daß er für die Fetthärtung überhaupt nicht brauchbar sei; diese Schlußfolgerung ist aber durchaus unrichtig.

Außer dem Nickelsulfat werden auch andere Nickelsalze, wie das Nitrat und das Chlorid, als Ausgangsstoffe genannt. Ob sie tatsächlich Anwendung finden, ist nicht sicher; es scheint beim Nitrat in beschränktem Maße der Fall zu sein. Angew. Chemie 1922. Nr. 63
Bald lernte man, die Wirkung des Nickels auch dadurch zu steigern, daß man mit ihm zugleich aus seinen Lösungen andere Stoffe, wie Kieselsäure, Tonerde oder dergleichen niederschlug.

Eine andere Gruppe von Patenten beschäftigt sich mit der Herstellung von Katalysatoren durch Abscheidung des Nickels aus seiner gasförmigen Verbindung mit dem Kohlenoxyd. Man läß̉ hierzu das Nickel sich auf einem. Träger abscheiden oder auch ohne Träger direkt im 81 .

Damit kommen wir zu einer weiteren Gruppe von Katalysatoren, die sich in der Praxis ein großes Gebiet erobert haben; das sind solche, die ohne Träger direkt in dem zu härtenden öl hergestellt werden. Es können hierzu alle direkt reduzierbaren Nickelverbindungen dienen, seien es anorganische, seien es organische. Praktische Anwendung haben hauptsächlich das Carbonat, das Formiat und das Oleat gefunden. Die Reduktion dieser Verbindungen unter ol erfolgt ebenfalls durch Wasserstoff unter Erhitzen. Das uulgebende öl verhindert ein Zusammensintern der freiwerdenden Metallteilchen, so daß sich das Nickel in einem fast kolloidal feinen Zustande im ol zu einer tintenschwarzen Flüssigkeit verteilt. Die Herstellung des Katalysators im öl und die daran anschlieBende eigentliche Härtung können in e in em Arbeitsgang durchgeführt werden. Die Hinzufügung eines Trügers ist überflüssig, es seí denn, daß man das Filtrieren des fertig gehärteten oles dadurch erleichtern will.

In dieser Gruppe von Katalysatoren ist fernerhin gefunden worden, $\mathrm{daB}$ es zur Reduktion der Nickelverbindungen nicht nötig ist, Wasserstoff einzuleiten. Das ameisensaure Nickel zerfällt schon an und für sich beim Erhitzen unter Lultabschluß unter Bildung freien Metalls. Aber auch andere Nickelverbindungen wie das Oxyd, das Carbonat und das Oleat gehen beim Erhitzen mit einem olubberschuß in katalysierendes Metall über. Merkwürdig erscheint nur, daß das ol selbst hierbei nicht in unangenehmer Weise verändert zu werden scheint; wenigstens sagen die Patentschriften nichts davon. Auch Kohlenwasserstoffe wie Paraffin werden zur Reduktion des Katalysators angewandt. Auch pulverförmige Kohle ist zur Reduktion von Nickelverbindungen zur Katalysatorherstellung vorgeschlagen.

Ein weiteres Verfahren ist von amerikanischer Seite auf Grund eines Versuches von Prof. Fre $\mathrm{rich}$ in Bonn ausgearbeitet worden. Dieses Verfahren geht von keiner Nickelver $\mathbf{b}$ ind ung, sondern von kompaktem käuflichen Nickelmetall aus. Durch Schleifen oder Mahlen des Nickels unter $\varnothing 1$ oder Wasser wird das Metall in den feinverteilten Zustand übergeführt. Ein solcher Katalysator arbeitet in der 'Tat besser als man eigentlich erwarten sollte. Das Verfahren hat jedenfalls den Vorzug, daß man außerordentlich wenig Sorgfalt bei ihm aufwenden muß. Man braucht weder auf eine bestimmte Temperatur, noch auf sorgfältigen Luftabschluß zu achten. Auch die Wiedergewinnung des toten Katalysators gestaltet sich zu einer sehr robusten, indem der tote Katalysator ohne Rücksicht auf organische Gifte einfach eingeschmolzen und danach von neuem gemahlen wird.

Die Ausführung des Härtungsverfahrens ist im allgemeinen grundsätzlich die gleiche geblieben wie zu Anfang. Sie besteht im innigen Durchmischen von $\delta 1$ und Katalysator mit Wasserstoff, was teils mit Hilfe von Rührwerken, teils durch Zerstäubung vorgenommen wird. Hierzu sind die verschiedenartigsten Apparate ersonnen worden, zum Teil von so phantastischer Art, daß sie eigentlich nur ein Kopfschütteln bewirken können. Mit diesen Apparaten geht es wieder wie mit den Katalysatoren: es läßt sich mehr oder weniger mit allen arbeiten. In Wirklichkeit geht aber die Reaktion, wenigstens bei einem guten $\delta 1$, so leicht vonstatten, daß selbst im einfachsten Apparat eine Beschleunigung nicht nur überflüssig ist, sondern daß man sie vielmehr häufig sogar künstlich mildern muß, um einen $\mathrm{zu}$ hohen Temperaturanstieg zu verhindern; denn die Reaktion findet unter Wärmeentwicklung statt.

Die für die Härtung angewandten Temperaturen sind verschieden. Kommt der Katalysator fertig in das öl, so wird die Reaktion gewöhnlich um $180^{\circ}$ herum vorgenommen. Wird der Katalysator im Ol selbst erzeugt, so ist hierzu eine höhere Temperatur erforderlich, gewöhnlich $230-250^{\circ}$. Hat sich der Katalysator im $\delta 1$ gebildet, so kann man mit der Härtung bei einer niedrigeren Temperatur fortfahren.

Die verschiedenen Verfahren arbeiten teils bei gewöhnlichem Atmosphärendruck, teils unter einem höheren Druck, in einem Falle sogar bei einem Druck von $50 \mathrm{Atm}$. und darüber.

\section{$\mathrm{W}$ is senschaftliches.}

In wissenschaftlicher Beziehung sind einige Arbeiten erschienen, die wohl über die Kreise der Härtungsfachleute hinaus Beachtung verdienen. Der alte Streit, ob das metallische Nickel oder ein niederes Oxyd desselben der eigentliche Katalysator sei, ist zur Ruhe gekommen, aber noch nicht endgültig entschieden. Da ich selbst am Streite der Meinungen früher lebhaft beteiligt gewe:en bin, will ich an dieser Stelle keine Ansicht über die einzelnen ArL eiten aussprechen, sondern nur sagen, daß ich bisher keinen AnsaB gefunden habe, von 
meiner alten Oberzeugung, daß das $M$ etall der Katalysator sei, abzugehen.

Eine gewisse theoretische Bedeutung hat das vorhin schon erwähnte Verfahren der Katalysatorherstellung durch Schleifen kompakten Nickelmetalls nach Frerichs. Da das so gewonnene feinverteilte Metall zufolge seiner Herstellung zweifellos regulinischer Natur ist, ist damit die gelegentlich vertretene Ansicht widerlegt, daß das katalytisch wirksame Nickel eine besondere Modifikation dieses Metalles sei. Ja, durch Pl a u s o n ist der Nachweis geführt, daß auch eine feine Verteilung des Metalls nicht unbedingt notwendig ist; die feine Verteilung kann durch eine außerordentlich innige Durchmischung ersetzt werden, wie sie in einer vernickelten $P$ l a i's o n schen Kolloidmühle erreichbar ist. Für die Praxis wird dieses Verfahren allerdings kaum in Betracht kommen.

Als Katalysatorgifte sind eine ganze Reihe von Stoffen erkannt worden, von denen diejenigen ein besonderes technisches Interesse haben, welche als Verunreinigungen in den angewandten Nickelsalzen vorkommen oder sonst im Verlaufe des Arbeitsganges in den Katalysator hineingelangen können. In dieser Beziehung sind besonders $B$ le $\mathrm{i}, \mathrm{Z}$ in $\mathrm{k}$ und $\mathrm{Sch}$ w ef $\mathrm{l}$ zu beachten. Die Halogene haben sich als erbeblich harmloser erwiesen, als seinerzeit von $S$ a b a $t$ i e $r$ angenommen wurde; die Nickelkatalyse ist geradezu mit herangezogen worden, um Halogene in organischen Verbindungen durch Wasserstolf zu ersetzen.

Das Kohlenoxyd, die häufige Verunreinigung des Wasserstoffs, wirkt, wenn ich mich so ausdrücken darf, auf den Katalysator weniger tötend als einschläfernd. Setze ich nämlich eine durch Kohlenoxyd gehemmte Katalyse mit reinem Wasserstoff fort, so erholt sich der Katalysator allmählich wieder.

Wenig erforscht sind die or $\mathrm{g}$ a $\mathrm{n}$ i s $\mathrm{ch}$ e $\mathrm{n} \mathrm{G}$ if $\mathrm{t}$ e, die in schlechten olen enthalten sind und sich oft sehr störend bemerkbar machen. Die Erforschung dieser Stoffe kann noch nützliche technische Erfolge mit sich bringen. Manche dieser Stoffe lassen sich auf mechanischem Wege durch Filtrieren mit den sog. Bleicherden entfernen; andere lassen sich durch Anwendung höherer Härtungstemperatur überwinden. Es scheint, daß sie durch die Katalyse bei höherer Temperatur in harmlosere Stoffe umgewandelt werden. Diese Annahme ist direkt bestätigt für das Kohlenoxyd, welches im wesentlichen erst oberhalb $230-250^{\circ}$ zu Methan reduziert wird ${ }^{1}$ ). Dieses Gas macht sich dementsprechend bei der für die Sättigung der Glyceride im allgemeinen günstigsten Temperatur von $170-180^{\circ}$ viel störender bemerkbar, als wenn man die Härtung aus irgendeinem Grunde bei einer höheren Temperatur vornimmt. Dies ist der Grund, warum in vielen Katalysator-Patenten behauptet werden konnte, daß der betr. Katalysator widersłandsfähiger gegen Gilte sci als andere. Im allgemeinen verhalten sich nach meinen Beobachtungen alle in Vorschlag gebrachten Formen der Katalysatoren den Giften gegenüber ähnlich.

Besondere Beachtung verdienen die Arbeiten von $R$ os e $n$ m und und $\mathrm{Z}$ e t $\mathrm{s} \mathrm{ch}$ e über die Beeinflussung der Katalysatoren durch gewisse Zusätze, welche bewirken, daß die Katalyse nicht bis zu dem theoretisch zu erwartenden Ende fortschreitet, sondern bei einer gewünschten $\mathrm{Z}$ wischenstufe haltmacht. Bisher haben die genannten Forscher ihre Untersuchung nicht auf die Fetthärtung ausgedehnt; es wäre aber sehr zu begrüßen, wenn das geschähe; denn die Härtungsindustrie sucht nach einem derartigen Katalysator. So wäre es z. B. erwünscht, wenn man die Sättigung der Linol- und Linolensäure zum Einhalt bringen könnte, wenn die Sältigungsstufe der Olsäure erreicht ist. Auf anderen Sondergebieten der Nickelkatalyse scheint eine derartige Abstimmung des Katalysators bereits mit Erfolg durchgeführt zu sein.

Daß die verschieden gestellten Doppelbindungen der Fettsäuren sich verschieden leicht sättigen lassen werden, ist von vornherein $\mathrm{zu}$ erwarten. Bestätigt ist dies durch eine Untersuchung von A r mstrong und Hilditch, welche fanden, daß eine Kurve, gezogen aus Zeit und Wasserstofiverbrauch bei der Härtung von Linolensäure zwei Knickpunkte aufweist an denjenigen Stellen, die einer Sättigung zu Linol- und Olsäure entsprechen. $\mathrm{M}$ o or $\mathrm{e}^{2}$ ) stellte fest, daß bei verhältnismäßig hoher Temperatur die übrigen Doppelbindungen ungesättigter Säuren sich leichter sättigen lassen als die 9-10-Bindung der ölsäure. Er gibt eine auf diese Beobachtung gegründete Arbeitsvorschrift, um die Bevorzugung gewisser Doppelbindungen in den Vordergrund treten zu lassen. Ob sich diese Vorschrift in der Praxis bewährt hat, ist mir nicht bekannt; in Amerika, wo derartige halbgehärtete Fette als Schmalzersatz sehr beliebt sind, sucht man dieses Ziel auf dem entgegengesetzten Wege zu. erreichen ${ }^{3}$ ). Eine grundsätzliche Lösung der Frage der auswählenden Sättigung scheint mir die Beobachtung von M o o r e auf keinen Fall zu bringen.

Eine andere Beobachtung von großem Interesse ist die, daß bel der Härtung Olsäuren entstehen, die in dem ursprünglichen $\not 1$ nicht vorhanden waren, wie Isoölsăure, Flaidinsäure oder auch bisher unbekannte Olsäuren. . Wir dürfen diese Erscheinung wohl nicht so verstehen, daß die Doppelbindung wandert, wie wir das von der Kalischmelze der Ölsäure nach $V$ a r r e $\mathrm{t} t \mathrm{r}$ a p her kennen; wabrscheinlicher ist schon, daB bei Anwesenheit mehrerer Doppelbindungen diese nicht der Reihe nach gesättigt werden, sondèrn daß diejenige Doppel-

1) Armstrong u. Hilditcb, Seifenfabr. 1920, S. 453.

2) Moore, Seifens.-Ztg.,1917, S. 651 .

3). Amer. Pat. 1135351 , จ. Burchenal. bindung zuerst angegriffen wird, die zufällig rein mechanisch zugleich mit Wasserstoff und einem Katalysatorteilchen in Berührung kommt. Auf diese Weise können aus der Linolensäure drei verschiedene Linolsäuren und drei ölsäuren nebeneinander entstehen.

Das Entstehen verschiedener Isomerer dürfte zum Teil auf diese Weise zu erklären sein; zum Teil kommt aber auch noch eine andere Erklärung in Betracht, nämlich eine Rückläufigkeit der Katalyse, die unter gewissen Bedingungen offenbar eintreten kann. Auch Moore zieht bei seinen oben mitgeteilten Versuchen eine solche in Betracht kann sie aber beim bloßen Zusammenbringen von Fett und Katalysator nicht feststellen "). Sie scheint aber doch vorzuliegen. Ich selbst habe im Laufe der Zeit wiederholt die Beobachtung gemacht, daß die Jodzahlen gegen Ende der Härtung wieder zunehmen statt bis auf Null herunterzugehen. Leider fand ich keine Zeit, diese interessante $\mathrm{Be}$ obachtung zu verfolgen. Neuerdings ist sie auch von amerikanischer Seite, von L e ve y, gemacht und in einer Patentschrift ${ }^{5}$ ) niedergelegt. Der Verfasser behandelt 01 mit Nickelkatalysator in einem Gasstrom bei $220-250^{\circ}$. Es erfolgt dabei eine Abspaltung von Wasserstoff und es entstehen aus nichttrocknenden Ölen unter Erhöhung der Jodzahl solche mit Trockeneigenschaften. Verschiedene Gase sind brauchbar, sogar Wasserstoff; die günstigsten Erfolge treten jedoch mit Kohlenoxyd ein.

Des weiteren findet derselbe Forscher, daß ein öI mit hoher Jodzahl, aber schlechter Trockeneigenschaft, in ein gut trocknendes $\delta$ übergeführt werden kann, wenn es erst teilweise mit Wasserstoff gesättigt und darauf nach seinem Verfahren wieder entwasserstofft wird. L e ve y schließt hieraus, daß die abgespaltenen Wasserstoffatome nicht dieselben sind wie die vorher angelagerten.

Die für diese Wasserstoffabspaltung angewandten Gase scheinen mehr mechanisch den abgespaltenen Wasserstoff zu entfernen, als chemisch wirksam zu sein. Nur bei dem am kräftigsten wirkenden Kohlenoxyd nimmt $L$ e v e y wohl mit Recht eine chemische Mitwirkung des Gases als Wasserstoff e m p fä $n$ g e $r$ unter Reduktion zu Methan an. Es wäre dies ein Seitenstück zu dem Verfahren von $K$ a y $\operatorname{ser}^{\circ}$ ) zur Härtung mit B o r n e ol statt mit Wasserstoff, wobei das Borneol unter Wasserstoffverlust in Kampher übergeht. Auch diese Beobachtung ist von großem Interesse, wenn sie auch kaum praktische Bedeutung haben dürfte. Einiges Interesse dürfte auch folgende Beobachtung bieten: Ich erwähnte vorhin, daß bei der Herstellung des Katalysators direkt im 81 dieser sich in Form einer tintenschwarzen Flüssigkeit löst. Diese Flïssigkeit läßt sich nur schwer filtrieren. Unterwerfe ich sie der Härtung, so flockt das Nickel nach ganzer oder teilweiser Sättigung des öles aus oder läßt sich doch leicht abfiltrieren. Hierau ist zuerst von $\mathrm{Erdm}$ a $\mathrm{n}$ aufmerksam gemacht worden. Vermische ich nun das abgesetzte oder abfiltrierte Nickel mit frischem 81 , so bekomme ich wieder die tintenschwarze Lösung. Vermische ich djeses Nickel mit Petroläther, so läßt sich die Mischung leicht filtrieren; vermische ich es aber mit Benzol, so bekomme ich wieder eine nicht- oder schwerfiltrierbare schwarzbraune Flüssigkeit. Es scheint somit, daß bei dieser Art von kolloidaler Lösung des Nickels die Doppelbindungen des Lösungsmittels eine Rolle spielen.

Der Gedanke, die Wasserstoffanlagerung nicht, wie Sabati e es tat, im Gas- und Dampfzustande, sondern im flüssigen Zustande des zu sättigenden Stoffes vorzumehmen, hat sich nicht nur in der Fetthärtung als erfolgreich erwiesen, sondern ist weit über diese hinaus angewandt worden. Ich will von allen nur an diejenige Anwendung erinnern, die nächst der Fetthärtung wohl am bekanntesten geworden ist, an die Sättigung des Naphthalins, die Tetralinfabrikation.

$$
\text { Wirtschaft I iches. }
$$

Besonders reizvoll ist es, nachzuforschen, welche wirtschaftliche Bedeutung die Fetthärtung im Laufe der Jahre erlangt hat. Leider läßt sich diese weniger klar übersehen als die technische und wissenschaftliche Entwicklung. Irgendwelche Angaben über die Welterzeugung an gehärteten Fetten liegen nicht vor. Klare Auskünfte von Firmen sind begreiflicherweise schwer zu bekommen und sind dann immer nur Einzelauskünfte, die sich nicht zu einem geschlossenen Gesamtbilde zusammenfügen lassen. Einige Firmen, denen ich auch an dieser Stelle meinen Dank aussprechen möchte, sind mir in sehr liehenswürdiger und bereitwilliger Weise entgegengekommen, so daß es mir vielleicht golingt, wenigstens ein paar Ausschnitte aus dem Gesamtbilde des gegenwärtigen Standes der wirtschaftlichen Entwicklung zu geben.

Die Fethärtung hat sich in dem verhältnismäBig kurzen Zeitraum von etwa 15 Jahren als eine eigene selbständige Industrie über die ganze Erde verbreitet. Es gibt kaum noch ein Kulturland, in dem keine Härtungsfabrik vorhanden wäre. Die erste Anlage, die labrikmäßig arbeitete, war bei der Firma $C$ ros $f$ i eld in England. Diese konnte im Jahre 1907 täglich etwa $2000 \mathrm{~kg}$ leisten. Die zweite Anlage, die erste auf dem Kontinent, war nicht, wie ich im Schrifttum gefunden habe, 1910 oder 1911 bei der Firma $\mathrm{Sch} \mathrm{icht} \mathrm{in} \mathrm{Aussig}$ sondern im Jahre 1908 bei der Firma Leprince \& Siveke in Herford in Tätigkeit, aus deren Laboratorium die Fetthärtung hervorgegangen war. Die dritte Anlage wurde im Jahre 1910 von W i l- 
buschewitsch in Nischni-Nowgorod nach Herforder Vorbild in Betrieb gesetzt.

Im Jahre 1914 sollen sehon im ganzen 24 Härtungsfabriken bestanden haben, davon 18 in Europa, von diesen 6 in Deutschland, mit einer Jahreserzeugung von 200-230 Millionen $\mathrm{kg}$ Fett. Jetzt stelle ich in Deutschland 11 Anlagen fest, die bedauerlicherweise zum überwiegenden Teile in ausländischen Händen sind. $\mathrm{Ob}$ diese Ziffer aber ausreicht, ist zweifelhaft; denn es sind unter den genannten 11 Fabriken schon mehrere enthaiten, die in den Zeitschriften nicht genannt sind, die ich vielmehr durch Erkundigungen in Erfahrung gebracht labe. Es scheint, daß es noch einige gibt, von denen überhaupt keine Nachricht in die weitere Offentlichkeit gedrungen ist. In erhöhtem Maße gilt dieser Zweifel an der Vollständigkeit der Zahlen natürlich für das Ausland.

Ich fand für europäische Länder, außer Deutschland, etwa 50 Fabriken,

$\begin{array}{ll}\text { in Holland } & \mathbf{5}-\mathbf{6} \\ \text { Belgien } & 2 \\ \text { Frankreich } & \mathbf{5} \\ \text { England } & \mathbf{3} \\ \text { Dänemark } & \mathbf{3} \\ \text { Norwegen } & \mathbf{4} \\ \text { Schweden } & 4 \\ \text { Rußland } & 2 \\ \text { Böhmen } & \mathbf{2} \\ \text { Ungarn } & \mathbf{5} \text {. wo }\end{array}$

teilung nur eine in Betrieb sein soll in Italien sollen vor dem Kriege mehrere im Bau gewesen sein,

in Rumänien, in der Levante, Osterreich, Schweiz, Spanien je 1 .

Von außereuropäischen Ländern

in der Mandschurei 1

$$
\begin{array}{ll}
\text { Agypten } & \mathbf{1} \\
\text { Japan } & 8 \text { Fabriken. }
\end{array}
$$

Das sind einschließlich Deutschland etwa 60 Fabriken, welche Zahl wohl als eine untere Grenze anzusehen ist, selbst wenn einige der Anlagen infolge der durch den Krieg veränderten wirtschaftlichen Verhältnisse den Betrieb gar nicht aufgenommen oder wieder eingestellt haben sollten.

Hierzu kommen noch 14-15 Fabriken in den Vereinigten Staaten von Amerika.

Als Rohstoffe sind und werden für die Härtung wohl sämtliche ole herangezogen, die überhaupt in technischem Maßstabe gewonnen werden. Sogar das Chrysalidenöl, das $O l$, welches aus den in den Seidenspinnereien abgetöteten Puppen des Seidenspinners gewonnen wird, scheint technisch gehärtet zu werden.

Die Eignung und Verwendung der gehärteten Fette zur Seifenfabrikation, die anfangs manches Vorurteil zú überwinden hatte, ist jetzt wohl so allgemein anerkannt und durchgeführt, daB ich keine Worte weiter darüber zu verlieren brauche.

Am meisten interessiert uns die Nutzbarmachung der Fethärtung für die menschliche Ernährung. Anfängliche Bedenken gegen die Verwendung gehärteter Fette für die menschliche Ernährung sind durch eine Reihe von Untersuchungen zerstreut worden. Nicht nur die Verdaulichkeit und Bekömmlichkeit an sich, sondern auch die genügende Ausnutzung der Hartfette im Körper wurde festgestellt, so daß der Weg für sie in die Industrie der Speisefette frei war. Bei uns wandern die g€bärteten Speisefette wohl durchweg in die Margarine; in Amerika sind sie aber auch für sich in sehmalzartiger Form als Schmalzersatz sehr beliebt.

Die vorher erwähnten 14-15 amerikanischen Fabriken sollen eine Leistungs $\mathrm{f} a \mathrm{~h} \mathrm{ig} \mathrm{k}$ it von $142000 \mathrm{t}$ im Jahre besitzen. Die wirkliche Leistung ist aber in den letzten Jahren nur elwa halb so grob gewesen; ich habe daruber folgende Zahlen nachträglich erhalten (in Tonnen zu je $1000 \mathrm{~kg}$ ):

Erzeugung.

\begin{tabular}{|c|c|c|c|c|c|}
\hline Jahr & Insgesamt & $\begin{array}{l}\text { 1. Jan. bis } \\
\text { 31. März }\end{array}$ & $\begin{array}{l}\text { 1. April bis } \\
30 \text {. Juni }\end{array}$ & $\begin{array}{l}\text { 1. Jali bis } \\
\text { 30. Sept. }\end{array}$ & $\begin{array}{l}\text { 1. Okt. bis } \\
\text { 31. Dez. }\end{array}$ \\
\hline \multicolumn{6}{|c|}{ Erzengung } \\
\hline $\begin{array}{l}1919 \\
1920 \\
1921\end{array}$ & $\begin{array}{l}54635 \\
8 t \quad 891 \\
98071\end{array}$ & $\begin{array}{r}4631 \\
19119 \\
19234\end{array}$ & $\begin{array}{r}8148 \\
11315 \\
23927\end{array}$ & $\begin{array}{r}9758 \\
23136 \\
28986\end{array}$ & $\begin{array}{l}32093 \\
28320 \\
52025\end{array}$ \\
\hline \multicolumn{6}{|c|}{ Verbrauch } \\
\hline $\begin{array}{l}1919 \\
1920 \\
1921\end{array}$ & $\begin{array}{l}24308 \\
16930 \\
26888\end{array}$ & $\begin{array}{r}3521 \\
3819 \\
6172\end{array}$ & $\begin{array}{l}6857 \\
2805 \\
6373\end{array}$ & $\begin{array}{l}6767 \\
4924 \\
8799\end{array}$ & $\begin{array}{l}7173 \\
4382 \\
5544\end{array}$ \\
\hline \multicolumn{6}{|c|}{ Vorráte } \\
\hline $\begin{array}{l}1919 \\
1920 \\
1921\end{array}$ & - & $\begin{array}{r}3534 \\
10543 \\
14601\end{array}$ & $\begin{array}{r}5931 \\
6755 \\
13186\end{array}$ & $\begin{array}{l}5859 \\
7024 \\
8775\end{array}$ & $\begin{array}{r}7828 \\
8469 \\
11632\end{array}$ \\
\hline \multicolumn{6}{|c|}{ Ausfuhr } \\
\hline $\begin{array}{l}1919 \\
1920 \\
1921\end{array}$ & $\begin{array}{r}1886 \\
821 \\
1068\end{array}$ & $\begin{array}{r}12 \\
372 \\
463\end{array}$ & $\begin{array}{l}165 \\
249 \\
171\end{array}$ & $\begin{array}{r}808 \\
51 \\
244\end{array}$ & $\begin{array}{r}90 \\
150 \\
179\end{array}$ \\
\hline
\end{tabular}

Diese Fettmengen sind pflanzlichen Ursprungs; Tran scheint bisher in den Vereinigten Staaten von Amerika kaum zur Härtung herangezogen $z u$ sein, doch steht auch dort eine umfangreiche Tranhärtung bevor. Das Fett hat zum überwiegenden Teil als Speisefett, hauptsächlich als Schmalzersatz gedient. Zur Seifenherstellung werden nur sehr geringe Mengen benutzt, da tierische Fette beträchtlich billiger waren als gehärtete.

Diese für Amerika mitgeteilten Zahlen erscheinen für das große Land gering; denn die Leistungsfähigkeit wie die wirkliche Leistung des doch viel kleineren Deutschlands hat selbst in der Zeit der starken Fabrikationseinschränkung während des Krieges der oben mitgeteilten amerikanischen nicht nachgestanden. Durch freundliches Entgegenkommen des Kriegsausschusses für Öle und Fette bin ich in der Lage, sowohl diese mu zeigen, wie auch ein Bild machen zu können von der Bedeutung, welche die Fetthärtung während des Krieges für die deutsche Volksernährung gehabt hat.

Während der Zeit der Fettnot, als alle Fettstolle zum Wohl der Allgemeinheit beschlagnahmt waren, sind vom Kriegsausschuß die in der nachfolgenden Aufstellung im einzelnen angegebenen Mengen Fett und $O I$ der Margarineindustrie zugeteilt worden.

Fettmengen, welche der Margarineindustrie zugewiesen Im Jahre: 1915

1916

\begin{tabular}{|c|c|c|c|c|c|c|}
\hline & $\begin{array}{l}\text { Gehärtetes } \\
\text { Pflanzen öl }\end{array}$ & $\begin{array}{c}\text { Gehärteter } \\
\text { Tran }\end{array}$ & $\begin{array}{l}\text { Naturfefte } \\
\text { und -öle }\end{array}$ & $\begin{array}{l}\text { Gehärtetes } \\
\text { Pflanzenöl }\end{array}$ & $\begin{array}{c}\text { Gehärteter } \\
\text { Tran }\end{array}$ & $\begin{array}{l}\text { Naturfette } \\
\text { und -öle }\end{array}$ \\
\hline $\begin{array}{c}\text { Januar } \\
\text { Februar } \\
\text { März } \\
\text { April } \\
\text { Mai } \\
\text { Juni } \\
\text { Juli } \\
\text { August } \\
\text { September } \\
\text { Oktober } \\
\text { November } \\
\text { Dezember }\end{array}$ & $\begin{array}{r}3180 \\
- \\
85 \\
- \\
- \\
-\end{array}$ & $\begin{array}{c}- \\
- \\
600 \\
100 \\
- \\
980 \\
-\end{array}$ & $\begin{array}{c}3180 \\
- \\
2175 \\
320 \\
- \\
2020 \\
-\end{array}$ & $\begin{array}{c}958 \\
483 \\
304 \\
398 \\
170 \\
- \\
-\end{array}$ & $\begin{array}{r}1315 \\
1950 \\
1764 \\
\\
2745 \\
\\
15 \\
143 \\
154 \\
429 \\
308 \\
468 \\
403\end{array}$ & $\begin{array}{r}12188 \\
7699 \\
2919 \\
\\
5950 \\
1633 \\
2018 \\
1995 \\
1930 \\
2318 \\
2628 \\
2666\end{array}$ \\
\hline \multicolumn{4}{|c|}{$\begin{array}{c}\text { zusammen } 12640 \\
\text { Hartfett : Naturfett }=2: 3\end{array}$} & \multicolumn{3}{|c|}{$\begin{array}{l}\text { zusammen } 56922 \\
\text { Hartfett : Naturfett }=1: 3^{1} / \mathrm{z}\end{array}$} \\
\hline
\end{tabular}

Im Jahre: 1917 1918

\begin{tabular}{c|c|c|c|c|c|c}
\hline & $\begin{array}{c}\text { Gehärtetes } \\
\text { Pllanzenöl }\end{array}$ & $\begin{array}{c}\text { Gehärteter } \\
\text { Tran }\end{array}$ & $\begin{array}{c}\text { Naturfette } \\
\text { und -olo }\end{array}$ & $\begin{array}{c}\text { Gehärtetes } \\
\text { Ptlanzenöl }\end{array}$ & $\begin{array}{c}\text { Gehärteter } \\
\text { Tran }\end{array}$ & $\begin{array}{c}\text { Naturfette } \\
\text { und -öle }\end{array}$ \\
\hline Januar & 116 & 436 & 2753 & 1049 & - & 3448 \\
Februar & - & 112 & 2718 & 1118 & - & 3261 \\
März & - & 566 & 2511 & 1402 & - & 2516 \\
April & 36 & 511 & 2648 & 1048 & - & 2004 \\
Mai & 266 & 213 & 2585 & 835 & - & 1593 \\
Juni & 96 & 66 & 1523 & 504 & - & 1518 \\
Juli & 366 & - & 1302 & 442 & - & 1623 \\
August & 568 & - & 2626 & 1458 & - & 2219 \\
September & 1021 & - & 2089 & 2393 & - & 3157 \\
Oktober & 1539 & - & 2990 & 2016 & - & 3563 \\
November & 968 & - & 3508 & 1689 & - & 3231 \\
Dezember & $\mathbf{8 3 4}$ & - & 2626 & 1293 & - & 2305 \\
\hline
\end{tabular}

Im Jahre: 1919

\begin{tabular}{c|c|c}
\hline & $\begin{array}{c}\text { Gebärtetes } \\
\text { Fett }\end{array}$ & $\begin{array}{c}\text { Naturfette } \\
\text { und -öle }\end{array}$ \\
\hline Januar & 697 & 1716 \\
Februar & 1916 & 3075 \\
März & 2392 & 2307 \\
April & 2515 & 2720 \\
Mai & 3263 & 2809 \\
Juni & 5440 & 4562 \\
Juli & 7024 & 8121 \\
August & 3316 & 10774 \\
September & 1382 & 6608 \\
Oktober & 817 & 8197 \\
November & 365 & 9680 \\
Dezember & 1294 & 11763 \\
\hline \multicolumn{3}{|c}{ zusammen 102 753 } \\
\multicolumn{2}{|c}{30421} & 72332 \\
Hartfett : Naturfett $=1: 2 \%$
\end{tabular}


Die schlimmsten Notjahre waren 1916-1919, vor allem 1917 und 1918. Wir sehen aus der Zusammenstellung, daß während dieser Zeit der Anteil des gehärteten Fettes an der Margarine ein Fünftel bis sogar zu e in e m D rit t e l der Gesamtmenge betragen hat. Nun war aber die Sachlage nicht einfach so, daß man aus einem guten öl, etwa einem ohnehin schon als Speiseöl dienenden Baumwollensaatöl, ein gutes und genießbares Hartfett erzeugen konnte. Solche ole hatten wir im Kriege nur sehr wenige, für Härtungszwecke jedenfalls gar keine. Hätten wir sie gehabt, so hätten wir unsern Fetthunger zur Not viel einfacher mit den ölen direkt, ohne sie erst zu härten, stillen können, wie der Italiener vielfach oll zur Speisebereitung benutzt, wo unsere Hausfrauen Fett zu gebrauchen gewohnt sind. Wir hatten im Kriege nur solche ole für die Härtung, die wir an und für sich zur Ernährung wegen ihres Geruchs und Geschmacks nicht gebrauchen konnten. Ich nenne vor allen Dingen Tran, dann Leinöl, welches wir aus den von uns besetzten östlichen Gebieten erhielten, Getreidekeimöl, sehr schlechtes Rüböl und aus Rumänien fast schwarzes Rapskuchenöl, das ist der letzte Rest des in den ölkuchen beim Pressen zurückbleibenden öles, der noch durch Benzinextraktion herausgeholt wurde - alles ole, die sonst für alles mögliche, nur nicht für Nahrungszwecke verwendbar erscheinen.

$\mathrm{Da}$ nun bekanntlich durch die Härtung der eigentümliche unangenehme Geruch und Geschmack der öle so vollständig verändert wird, daß die erzeugten Fette nicht das geringste mehr von ihrem Ursprung verraten und infolgedessen als Speisefette brauchbar werden, wurde es auf diesem Wege möglich, die genannten ole für unsere Ernährung heranzuziehen und den doch recht geringen vorhandenen Vorrat an Nahrungsfetten b is u m di e Hälf te (im Jahre 1918) zu vermehren - ein recht beachtenswerter Beitrag zum Durchhalten in dem uns aufgezwungenen Kriege!

Gehärteter Tran als Speisefett ist aber nicht nur als eine Kriegserscheinung anzusehen; er ist vielmehr durchaus imstande, eine tadellose Margarine zu ergeben. Das zeigt uns Dänemark, das schon vor dem Kriege, im Jahre 1914, 3,4 Millionen kg gehärteten Waltran eingefühit und auf Margarine verarbeitet hat ${ }^{7}$ ). Es wird uns ausdrücklich versichert, daß sich der gehärtete Waltran ausgezeichnet zur Herstellung einer wohlschmeckenden und dauerhaften Margarine eignet.

Lobhaft beklagt wird die kräftige Entwicklung der Härtungsindustrie jedenfalls von den Walfischen und anderen Seetieren; denn diese sehen sich durch die Fanggesellschaften, die zugleich mit der Fetthärtung einen außerordentlichen Aufschwung genommen haben ernstlich in ihrem Dasein bedroht. Im Norden ist dieses Seewild schon fast ausgerottet; auf der südlichen Hälfte unseres Erdballs, wo jetzt die Hauptfangplätze liegen, wird dies auch in absehbarer Zeit der Fall sein, wenn nicht eine kraftvoll internationale Gesetzgebung eingreift. Aber selbst dann ist eine starke Verminderung der Tranerzeugung zu erwarten, und die Fetthärtung wird je lảnger je mehr auf Pflanzenöle angewiesen sein. Für diese aber, die uns von der Natur in weit reicherem Maße geboten werden als die festen Fette, eröffnet sich in der Härtung ein neues Absatzgebiet; denn sie können durch die Härtung in die viel wertvollere und höher geschäłzte feste und halbfeste Form übergeführt werden und dadurch den Weltvorrat an Nahrungsfetten vergrößern. Es ist also zu erwarten, daß der olanbau eine Belebung erfährt; und aus den Zeitungsnachrichten über die Gründung neuer Anbaugesellschaften zu schließen, ist das auch schon der Fall. Ob es vorteilhaft sein wird, in unserm Vaterlande jetzt OIsaaten an Stelle anderer Nahrungsmittel anzubauen, ist ein landwirtschaftliches Rechenexempel, dessen Beurteilung sich dem Chemiker entzieht. Auf alle Fälle aber würde es eine dankbare Aufgabe für die a $\mathrm{ng}$ e w a n d t e B o $t$ a $n$ ik sein, den olertrag der heimischen Saaten durch Züchtung zu steigern. Ein Beispiel, daß derartiges möglich ist, bietet uns die Zuckerrübe, bei welcher es durch geeignete Züchtung gelungen ist, den Zuckerertrag auf das Zehnfache zu bringen.

Wie weit wir von der Entwicklungsgrenze noch entfernt sind, an der die Rentabilität der Härtung aufhört, ist schwer zu sagen. In Amerika scheint eine solche zur Zeit erreicht zu sein; denn mein amerikanischer Gewährsmann, der als einer der besten Kenner der Härtungsindustrie der Vereinigten Staaten gilt, schreibt darüber wie folgt:

„Seit dem Jahre 1918 ist es nicht sehr profitabel gewesen, öle zu härten, weder für Speisezwecke noch für die Seifenerzeugung. Als Grund dafür möchte ich die Tatsache erwähnen, daß die Härtung eine so einfache und leicht verständliche Sache geworden ist, daß der Preis von $O \mathrm{l} \mathrm{zu}$ einer solchen Höhe gestiegen ist, daß nur nominale Herstellungskosten berechnet werden dürfen, um im Preise mit den natürlichen harten Fetten konkurrieren zu können, für welche die gehärteten olle substituiert werden. Von den genannten 14 Fabriken sind die letzten drei seit 1918 außer Betrieb. Sie arbeiteten in Lohn und konnten keine gewinnbringende Beschäftigung tinden, trotzdem die beiden elektrischen Anlagen in Niagara Falls wegen ihrer billigen elektrischen Kraft in einer ausgezeichneten Lage waren, um mit sehr geringen Kosten härten zu können."

Diese amerikanische Schilderung braucht uns aber nicht zu schrecken, und es ist kein Grund, warum wir den augenblicklichen Zustand als einen dauernden ansehen sollten; denn wir erkennen in der amerikanischen Zahlenreihe ein ertreuliches starkes Wiederansteigen der Härtungstätigkeit. Auch bei uns haben wir früher vorübergehend schon Preisverhältnisse auf dem Fettmarkte gehabt, bei denen ein Nutzen der Härtung nahezu oder auch ganz aufgehoben war. Eine solche Marktlage war z. B. ein wesentlicher Grund, warum die Fetthärtung in den ersten Jahren nach ihrer Erfindung nicht sofort festen Fuß fassen konnte. Wie wir gesehen haben, hat sich bisher die Marktlage immer wieder in der Weise geändert, daß die Härtungsindustrie sich mit Erfolg durchsetzen konnte. Wie aber die Rentabilität sich auch gestalten möge: ihren volkswirtschaftlichen Wert als machtvoller Regulator des Preisverhältnisses zwischen flüssigen ölen und festen Fetten wie durch Vergrößerung des Weltvorrats an Nahrungsfetten wird die Härtungsindustrie für absehbare Zeit behalten und nicht eher wieder verlieren, als bis das Gute, das sie uns gebracht hat, durch efwas Besseres abgelöst wird. Bis dahin aber wollen wir ihr eine kräftige und gesunde Weiterentwicklung wünschen zum Segen unseres bedrückten Vaterlandes.

[A. 156.]

\section{Untersuchungen über künstlichen und natür- lichen Graphit.}

\author{
Von K. ARNDT und F. KöRnER, Charlottenburg. \\ (Eingeg. 27.15. 1922.)
}

Für den Elektrochemiker ist neben der chemischen Widerstandsfähigkeit die gute elektrische Leitfähigkeit die wichtigste Eigenschaft des Grapbits. In der Literatur ist hierüber wenig zu finden. In Landolt-Börnsteins Tabellen ist für einige natürliche Graphite die Leitfăhigkeit angegeben; aber die Zahlen weichen sehr voneinander ab, und es ist nicht gesagt, ob sie sich auf pulverförmigen oder festen Graphit beziehen. Auch die Einsicht in die Abhandlungen selbst liefert nur eine unbefriedigende Ausbeute. Über den künstlichen, im elektrischen Widerstandsofen nach dem Achesonverfahren hergestellten Graphit ist aus dem fast 20 Jahre alten, aber immer noch recht brauchbaren Büchlein von Fitz-Gerald und aus der Fachliteratur einiges wenige zu entnehmen.

Vor sechs Jahren ') hat der eine von uns einen Apparat beschrieben, um den eleklrischen Widerstand von Pulvern unter bestimmtem Druck zu messen. Dieser Apparat besteht aus einer Hebelpresse, mit der das in einem dickwandigen Porzellanrohr befindliche Pulver zwischen zwei Messingstempeln gepreßt wird; die Stempel dienen zugleich als Stromzufübrungen. Fig. 1 zeigt diese Anordnung. Der Widerstand der zwischen den Stempeln befindlichen Graphitsäule von $0,4 \mathrm{gcm}$

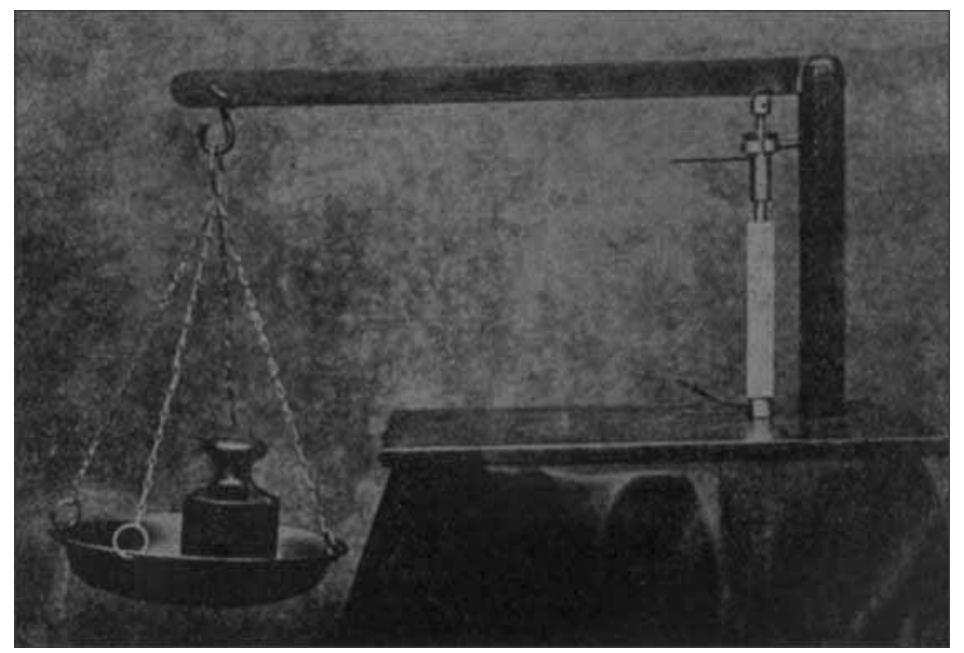

Fig. 1.

Querschnitt und etwa $5 \mathrm{~cm}$ Höhe wird mit der Wheatestoneschen Brbicke gemessen. Weil für die guten Graphite dieser Widerstand nur einige Zehntel $\mathrm{Ohm}$ beträgt, muß der Widerstand der Zuleitungen genau ermittelt und in Rechnung gesetzt werden. Der Ubergangswiderstand von den Stempeln zum Pulver, der bei geringeren Drucken großen Einfluß hat und wegen seines Sthwankens genwue Messungen autserordentlich erschwert, ist bei Drucken tuber $100 \mathrm{Atm}$. gering und bei dem Drucke von 175 Atm., unter dem wir die Widerstände maßen, praktisch zu vernachlässigen.

Beim Einbringen des Pulvers muß man mit einem Drabt storhern, damit sich nicht Luft zwischen die Teilchen lagert und einen zu hohen Widerstand vortäuscht. Bei Beobachtung dieser Vorsicht erhält man für jede Substanz sichere Werte, die allerdings sehr wesentlich von der GröBe der Pulverteilchen aubängen. Die folgende Tabelle gibt für einige der von uns untersuchten Graphite den elektrischen Widerstand, bezogen auf eine Säule V(ın $1 \mathrm{~m}$ Lănge und $1 \mathrm{qmm}$ Querschnitt, ferner den Aschengehalt und die mittlere TeilchengrbBe.

1) Zeitschr. S. Elektrochemie, Bd. 23, 176 [1917].

7) Chem. Ztg. 1919, S. 747. 